\title{
Elaboração e validação de uma tecnologia educacional acerca da violência contra a mulher
}

\author{
Elaboration and validation of an educational technology related to violence against women \\ Elaboración y validación de una tecnología educacional sobre violencia contra las mujeres
}

\begin{abstract}
Elayne Kelly Sepedro Sousa ${ }^{1}$ (C)
Erica Jorgiana dos Santos de Morais ${ }^{1}$ (B) Fernanda Cláudia Miranda Amorim ${ }^{1}$ (1)

Adélia Dalva da Silva Oliveira ${ }^{1}$ (1)

Kayo Henrique Jardel Feitosa Sousa ${ }^{2}$ (1)

Camila Aparecida Pinheiro Landim Almeida ${ }^{3}$ (1)
\end{abstract}

1. Centro Universitário UNINOVAFAPI.

Teresina, PI, Brasil.

2. Universidade Federal do Rio de Janeiro, Escola de Enfermagem Anna Nery. Rio de Janeiro, RJ, Brasil.

3. Universidade Católica Portuguesa, Instituto de Ciências da Saúde. Porto, Portugal.

\begin{abstract}
RESUMO
Objetivo: elaborar e validar uma tecnologia educacional acerca da violência contra a mulher. Método: estudo metodológico de validação de conteúdo e aparência. Para a validação, participaram deste estudo 11 especialistas de conteúdo e técnicos, 7 especialistas de design e 79 mulheres. Foram utilizados três instrumentos para a coleta de dados, um para cada grupo de participantes, e os dados foram analisados quanto ao nível de concordância e Índice de Validade de Conteúdo. Resultados: a média de concordância dos especialistas de conteúdo e técnicos foi de $87 \%$, o Índice de Validade de Conteúdo global por parte dos especialistas de design foi de 0,92 e o nível de concordância das respostas positivas pelo público-alvo foi de $96,2 \%$. Conclusão e implicações para a prática: o Alertômetro mostrou-se uma tecnologia educacional válida para ser utilizada, pois promove conhecimento acerca das diversas manifestações da violência contra a mulher.
\end{abstract}

Palavras-chave: Violência contra a Mulher; Tecnologia Educacional; Estudos de Validação; Educação em Saúde.

\begin{abstract}
Objective: to develop and validate educational technology about violence against women. Method: methodological study of content and appearance validation. For validation, 11 content specialists and technicians, 7 design specialists and 79 women participated in this study. Three instruments were used for data collection, one for each group of participants, and the data were analyzed for level of agreement and Content Validity Index. Results: the average agreement of the content specialists and technicians was $87 \%$, the global Content Validity Index by the design specialists was 0.92 and the level of agreement of the positive responses by the target audience was $96,2 \%$. Conclusion and implications for practice: the "Alertômetro" proved to be a valid educational technology to be used, as it promotes knowledge about the various manifestations of violence against women
\end{abstract}

Keywords: Violence against Women; Educational technology; Validation Studies; Health education.

\section{RESUMEN}

Objetivo: desarrollar y validar tecnología educativa sobre la violencia contra la mujer. Método: estudio metodológico del contenido y validación de la apariencia. Para la validación, 11 especialistas y técnicos de contenido, 7 especialistas en diseño y 79 mujeres participaron en este estudio. Se utilizaron tres instrumentos para la recopilación de datos, uno para cada grupo de participantes, y los datos se analizaron para determinar el nivel de acuerdo y el Índice de validez del contenido. Resultados: el acuerdo promedio de los especialistas y técnicos de contenido fue del $87 \%$, el índice de validez de contenido global por parte de los especialistas en diseño fue de $0.92 \mathrm{y}$ el nivel de acuerdo de las respuestas positivas por parte del público objetivo fue de 96 2\% Conclusión e implicaciones para la práctica: el "Alertômetro" demostró ser una tecnología educativa válida para ser utilizada, ya que promueve el conocimiento sobre las diversas manifestaciones de violencia contra las mujeres.

Palabras clave: Violencia contra la Mujer; Tecnología Educativa; Estudios de Validación; Educación en Salud.
Autor correspondente:

Kayo Henrique Jardel Feitosa Sousa

E-mail: kayohenriquejardel@hotmail.com

Recebido em 11/11/2019.

Aprovado em 15/03/2020.

DOI: 10.1590/2177-9465-EAN-2019-0314 


\section{INTRODUÇÃO}

A violência contra a mulher está associada às diferenças entre os sexos em um relacionamento, e a naturalização dessas diferenças é fruto de uma construção cultural e social imposta, estabelecendo-se, a partir dela, relações de dominação e violência. ${ }^{1,2}$ Por conta disso, manifesta-se a necessidade de que a prática dos profissionais de saúde esteja pautada no objetivo de transformar valores e costumes hegemonicamente construídos, defendidos e aceitos pela sociedade, contribuindo para atitudes de desnaturalização. ${ }^{3}$

Corroborando com a ideia da naturalização da violência contra a mulher, um estudo realizado em 49 países evidenciou que aqueles com baixa e média renda apresentaram maiores taxas de aceitação social da violência. ${ }^{4} \mathrm{Na}$ Tailândia, 2.462 mulheres casadas foram entrevistadas sobre suas experiências em relacionamentos, constatando-se que 1 em cada 6 enfrentaram a violência. Estas relataram ainda que, nos últimos 12 meses, a violência psicológica foi a mais comum (60-68\%), seguida por violência sexual (62-63\%) e física (52-65\%). ${ }^{5}$

O profissional de saúde pode atuar em situações como essas ao tentar reduzir o ciclo da violência, evitando que casos simples se tornem mais graves. Para isso, ele deve conhecer a articulação da rede de apoio e promover ações de educação permanente em saúde, visando esclarecimentos e construção de vínculos com sua comunidade. ${ }^{6}$

Durante a prática profissional, o uso de instrumentos capazes de identificar e conduzir situações de violência facilita a atuação dos diversos profissionais de saúde, que têm a oportunidade de atender as mulheres que recorrem aos serviços em busca de ajuda com necessidades imediatas, porventura ligadas às condições de violências sofridas. ${ }^{7}$

As tecnologias educacionais podem ser utilizadas como suporte no processo de ensino-aprendizagem por apresentar informações atuais com evidências clínicas. Desta feita, considera-se importante, na elaboração de materiais educativos, a interdisciplinaridade dos conhecimentos que se complementam e tornam a tecnologia mais atrativa, utilizando os pilares de ensino e o conhecimento científico.

Os quatro pilares de ensino contribuem para a construção de tecnologias com o objetivo de auxiliar na formação holística do indivíduo. "Aprender a conhecer" indica o interesse, o despertar da curiosidade intelectual, a formação de um senso crítico; "aprender a fazer" mostra o processo de execução e o trabalho coletivo, com base nas relações interpessoais; "aprender a conviver" desenvolve a percepção de compreender o outro, participar de projetos de compreensão; e, finalmente, "aprender a ser", apresenta o desenvolvimento integral da pessoa e o pensamento autônomo e crítico. ${ }^{8}$

Nesse sentido, a tecnologia educacional proposta neste estudo surge como uma oportunidade de ressignificação das ações educativas referentes à violência contra a mulher. Quando aplicada, ela propiciará uma reflexão tanto do profissional, quanto da mulher, desmistificando a falsa imagem de que a violência é apenas física e reconhecida somente quando deixa marcas na vítima. Permitirá à mulher, ainda, a capacidade de analisar os atos cometidos por seus parceiros e refletir sobre as manifestações da violência que estes já tenham cometido ou estejam na iminência de cometer.

Ao considerar os aspectos citados, objetivou-se, neste estudo, elaborar e validar uma tecnologia educacional acerca da violência contra a mulher. Com isso, almeja-se contribuir para a prevenção da violência contra a mulher, proporcionando conhecimento e auxiliando os profissionais nas ações de educação em saúde com o público feminino.

\section{MÉTODO}

Trata-se de um estudo metodológico que consiste em uma pesquisa relacionada às investigações dos métodos de obtenção, organização e análise dos dados, descrevendo de forma pormenorizada a elaboração e análise dos instrumentos e técnicas de pesquisa. Este, por sua vez, objetiva a construção de um instrumento que seja confiável, preciso e utilizável, podendo ser aplicado por outros pesquisadores. ${ }^{9}$

Inicialmente, foi realizada a fase de elaboração do material, com uma revisão integrativa das publicações disponíveis sobre o tema. Na fase de análise, entre os meses de junho e setembro de 2018, tal conhecimento foi utilizado para construção do conteúdo teórico abordado.

Utilizou-se como bases de dados na revisão integrativa da literatura: Medical Literature Analysis and Retrieval System Online (MEDLINE), Literatura Latino-Americana e do Caribe em Ciências da Saúde (LILACS) e Base de Dados de Enfermagem (BDENF). Utilizaram-se os descritores MesH Women, Battered Women, Domestic Violence e Intimate Partner Violence para a busca na MEDLINE, e os descritores controlados Mulheres, Mulheres Agredidas, Violência contra a Mulher, Violência Doméstica e Violência por Parceiro Íntimo para a LILACS e BDENF, combinados com os operadores boleanos AND e OR.

Foram incluídos artigos que elencassem os fatores de risco da violência contra a mulher publicados entre 2013 e 2017, nos idiomas inglês, português e espanhol, e excluídos relatos de casos informais, capítulos de livros, dissertações, teses, reportagens, notícias, editoriais, textos não científicos e artigos sem disponibilidade do texto na íntegra on-line. A busca dos artigos foi realizada no mês de maio de 2018 e, em seguida, com o auxílio de um designer gráfico, a tecnologia educacional foi elaborada, utilizando-se o programa CorelDraw 2017.

Para a validação, selecionaram-se dois grupos de avaliadores, compostos tanto por especialistas de conteúdo e técnicos - que analisaram o conteúdo, a linguagem, a apresentação, a estimulação e motivação e adequação cultural -, quanto por especialistas de design - para validar a aparência. Esse processo ocorreu durante os meses de junho a agosto de 2018.

Para análise do Alertômetro, o levantamento da elegibilidade dos participantes para a função de especialistas de conteúdo e técnicos e especialistas de design foi realizado por meio da Plataforma Lattes e pela amostragem do tipo snowball sampling. Nela, o participante selecionado indica ou sugere outros 
participantes - técnica bastante utilizada quando a população é composta por pessoas com características difíceis de serem encontradas. ${ }^{10}$

A seleção se deu da seguinte forma: após acessar o site "Plataforma Lattes", na janela "Currículo Lattes", escolheu-se a opção "Buscar currículo". Na aba de busca avançada por assunto, foram usadas as palavras-chave: "Tecnologias Educativas", "Elaboração e Validação de Materiais Educativos" e/ou "Violência Contra a Mulher".

Estabeleceu-se, como parâmetro para a escolha de profissionais, o sistema de classificação de juízes, proposto em uma dissertação de mestrado em Fortaleza-CE e adaptado pelos autores deste estudo, adequando-se, assim, aos objetivos propostos pela presente pesquisa. Foram selecionados aqueles que obtiveram minimamente cinco pontos, em consonância com estudos de validação realizados anteriormente, ${ }^{11-13}$ conforme critérios de pontuação.

O público-alvo também foi consultado. Obteve-se, em setembro de 2018, uma população de 227 mulheres registradas nas zonas regionais, com dados levantados através do Sistema de Cadastramento de Usuários do Sistema Único de Saúde (CAD-SUS), em Teresina-PI. O tamanho da amostra foi definido por uma fórmula matemática específica, cujo objetivo foi estimar o tamanho mínimo necessário para o desenvolvimento de procedimentos estatísticos específicos, garantindo a confiabilidade do estudo. ${ }^{14,15}$

Utilizou-se amostragem do tipo aleatória simples com reposição. A Região Norte da cidade foi escolhida para a pesquisa por se tratar da maior zona populacional, haja vista que, na maioria das vezes, a violência é maior em áreas de grande densidade demográfica.

Após a realização do cálculo amostral, chegou-se ao quantitativo de 79 participantes. Foram considerados critérios de inclusão para o estudo: ser mulher adulta, na faixa etária compreendida entre 18 e 59 anos, e estar em atendimento nas Unidades Básicas de Saúde (UBS). Foram excluídas aquelas com algum tipo de distúrbio cognitivo e/ou mental, autodeclarado ou informado por acompanhante.

Por se tratar de uma análise utilizando grupos amostrais, julgou-se necessária a utilização de três instrumentos para a coleta de dados: o primeiro direcionado aos especialistas de conteúdo e técnicos, o segundo aos especialistas de design e o terceiro ao público-alvo.

Para os especialistas de conteúdo e técnicos, utilizou-se o Suitability Assessment of Materials (SAM), que avalia a dificuldade e conveniência dos materiais educativos, sendo analisada a concordância e relevância de cada item ( 1 = inadequado, 2 = parcialmente adequado e 3 = adequado). ${ }^{16} \mathrm{O}$ instrumento destinado aos especialistas de design era semelhante quanto à estrutura, contando também com escala do tipo Likert, mas com a seguinte valoração: 1 = discordo totalmente, 2 = discordo parcialmente, 3 = concordo e 4 = concordo totalmente, no que se refere à validação da aparência.
Para o público-alvo, porém, utilizou-se um questionário de avaliação adaptado ${ }^{16}$ ao participante, que melhor se adequava à linguagem das entrevistadas. Ele foi dividido em três partes: a primeira, com os aspectos sociodemográficos e econômicos a segunda, com os itens acerca dos domínios organização, estilo de escrita, aparência e motivação; e a terceira com um espaço aberto para as participantes emitirem suas opiniões.

As informações profissionais sobre os especialistas e os dados sociodemográficos do público-alvo foram processados no software Statistical Package for the Social Sciences (SPSS). A análise foi descritiva, por meio de frequência absoluta (n) e relativa (\%), quando se tratava de variável qualitativa. As variáveis quantitativas foram analisadas mediante as estatísticas de posição (média) e de variabilidade (desvio padrão).

Para a análise dos resultados dos especialistas de conteúdo e técnicos, efetuou-se o cálculo da porcentagem de escores obtidos por meio da soma total dos valores assinalados dividida pelo total de escores constantes no instrumento, sendo considerado valor igual ou superior a $60 \%$ como indicador de adequabilidade. ${ }^{17}$

Quanto à análise dos resultados dos especialistas em design, empregou-se a lógica do cálculo do Índice de Validade de Conteúdo (IVC), visto como adequado aos objetivos. O escore do item foi calculado por meio da soma de concordância dos itens assinalados por " 3 " ou "4", dividida pelo número total de respostas. Já o escore do instrumento foi realizado mediante a média da soma do IVC de cada item dividida pela quantidade de itens do instrumento. ${ }^{16} \mathrm{O}$ ponto de corte empregado para 0 IVC foi de $0,78 .^{18}$

O instrumento de coleta de dados para o público-alvo foi adaptado e construído em forma de escala, a fim de se obter uma medida mais objetiva da análise de cada um dos itens. Em relação à análise, adotou-se um nível mínimo de concordância de $75 \%$ das respostas positivas acerca da análise do material. ${ }^{13}$

O estudo foi aprovado por Comitê de Ética em Pesquisa, sob os Pareceres $n . \stackrel{0}{3}$ 3.023.137 e $n . \stackrel{0}{3}$ 3.023.147, e atendeu a todos os preceitos éticos para as pesquisas que envolvem seres humanos.

\section{RESULTADOS}

$\mathrm{Na}$ etapa de elaboração da tecnologia educacional, a revisão integrativa da literatura direcionou o conteúdo teórico a ser abordado, elencando as diversas manifestações da violência contra a mulher. Foram evidenciadas três formas de apresentação da violência, a saber: sexual, psicológica e física. As manifestações relacionadas foram extraídas dos 17 artigos incluídos na revisão.

Quanto à metodologia, selecionaram-se pesquisas de natureza qualitativa, quantitativa, quanti-qualitativa, estudos transversais e de coorte. Quanto aos tipos de violência, 15 artigos evidenciaram violência física, 12 violência psicológica e 9 a sexual.

A primeira versão da tecnologia foi idealizada pelos autores e construída com o auxílio de um designer gráfico. À medida que o profissional produzia as ilustrações, estas eram enviadas para 
aprovação ou possíveis alterações pelos pesquisadores, até que fosse alcançada a primeira versão da tecnologia educacional.

A primeira versão submetida à validação dos especialistas de conteúdo e técnicos e de design chamava-se "Violentômetro" e possuía o formato de um termômetro graduado. Cada grau do termômetro representava uma manifestação de um tipo de violência, dando a ideia de progressão da violência conforme o termômetro "esquentava". Ou seja, a temperatura do termômetro ficava mais alta quando a violência tomava proporções mais graves. Por exemplo: a menor temperatura, ou seja, $1^{\circ} \mathrm{C}$, representava piadas eróticas, e a maior temperatura, $60^{\circ} \mathrm{C}$, representava $\circ$ feminicídio (morte).

Os primeiros 15 graus do termômetro representavam a violência sexual, identificada pela cor amarela. O próximo nível, representado pela cor laranja, dizia respeito à violência psicológica,

Tabela 1. Validação dos especialistas de conteúdo e técnicos quanto ao conteúdo, linguagem, apresentação, estimulação/ motivação e adequação cultural. Teresina, Piauí, 2018

\begin{tabular}{cccc}
\hline Especialista & $\begin{array}{c}\text { Escore } \\
\text { obtido }\end{array}$ & $\begin{array}{c}\text { Possibilidade } \\
\text { total de escores }\end{array}$ & $\%$ \\
\hline E01 & 26 & 30 & 86,7 \\
\hline E02 & 28 & 30 & 93,3 \\
\hline E02 & 30 & 30 & 100 \\
\hline E04 & 26 & 30 & 86,7 \\
\hline E05 & 28 & 30 & 93,3 \\
\hline E06 & 28 & 30 & 93,3 \\
\hline E07 & 20 & 30 & 66,7 \\
\hline E08 & 26 & 30 & 86,7 \\
\hline E09 & 24 & 30 & 80 \\
\hline E10 & 30 & 30 & 100 \\
\hline E11 & 24 & 30 & 80 \\
\hline SAM GLOBAL & & & 87,9 \\
\hline
\end{tabular}

*SAM: Suitability Assessment of Materials dividida em 25 graus. $O$ último nível correspondia à violência física, sendo representado pela cor vermelha, contendo 20 graus.

$\mathrm{Na}$ etapa de validação, participaram 11 especialistas de conteúdo e técnicos, com idade média de $39( \pm 10)$ anos, predominantemente do sexo feminino (90,9\%). Quanto à formação, $81,8 \%$ eram enfermeiros, mas havia também uma cirurgiã dentista e uma advogada especializada em violência contra a mulher. Eram formados há mais de 10 anos, com média de 15( \pm 09$)$ anos. Quanto à titulação, mestres e doutores equivaleram-se numericamente, representando $45,4 \%$ cada.

A partir dos escores obtidos, a tecnologia educacional foi validada pelos especialistas de conteúdo e técnicos, mediante escore SAM global de 87,9\%, conforme a Tabela 1.

Compuseram a fase de validação da aparência sete especialistas em design. A caracterização da amostra revelou participantes jovens, entre 27 e 44 anos, com média de $35( \pm 6,1)$ anos, formados há $10( \pm 6,9)$ anos, predominantemente do sexo masculino (85,7\%) e atuantes em Teresina-PI (85,7\%).

Apenas um especialista de design discordou nos itens 1,2 e 3 . Por outro lado, nos itens 4 e 5 , todos concordaram que as orientações estão em locais estratégicos e que a tecnologia contribui para a mudança de comportamentos e atitudes, conforme a Tabela 2.

O IVC de cada item e o instrumento geral foram validados, pois o menor valor atribuído a um item foi 0,86 , e o maior, 1,0 . O IVC global foi de 0,92.

Após as contribuições dos especialistas que analisaram a tecnologia educacional, foram julgadas necessárias algumas adequações para que esta pudesse ser de fato utilizada junto ao público feminino, com o objetivo primordial de alertar sobre a violência contra a mulher. Dentre elas estava o nome da tecnologia, que passou de "Violentômetro" para "Alertômetro". A versão final recebeu esse nome pelo fato de alertar as mulheres sobre quais são as manifestações da violência, sem, no entanto, graduar por tipo e nível de gravidade. Graduar tornou-se inviável, uma vez que a violência é algo muito subjetivo, conforme a Figura 1.

Após as adequações, o processo de validação foi finalizado a partir das contribuições do público-alvo. Nessa etapa, participaram 79 mulheres, prevalecendo a faixa etária de 30 a 49 anos $(53,16 \%)$, pardas $(69,62 \%)$ e solteiras $(50,63 \%)$. Em relação à

Tabela 2. Análise dos especialistas da área de design quanto à caracterização da aparência. Teresina, Piauí, 2018

\begin{tabular}{|c|c|c|c|c|}
\hline Características da aparência & Discordo & Concordo & $\begin{array}{l}\text { Concordo } \\
\text { totalmente }\end{array}$ & IVC* \\
\hline $\begin{array}{l}\text { As frases organizadas em níveis de cores estão apropriadas } \\
\text { para chamarem a atenção do público-alvo. }\end{array}$ & 01 & 02 & 04 & 0,86 \\
\hline É clara e transmite facilidade de compreensão. & 01 & 01 & 05 & 0,86 \\
\hline As cores e formas estão adequadas para o tipo de material. & 01 & 04 & 02 & 0,86 \\
\hline As orientações estão em locais estratégicos para reflexão. & - & 05 & 02 & 1,0 \\
\hline $\begin{array}{l}\text { Contribui para a mudança de comportamentos e atitudes do } \\
\text { público-alvo. }\end{array}$ & - & 03 & 04 & 1,0 \\
\hline IVC GLOBAL & & & & 0,92 \\
\hline
\end{tabular}

*Índice de Validade de Conteúdo 
renda familiar, $61,5 \%$ referiam que o somatório dos rendimentos é menor que um salário mínimo, e, quanto à religião, 69,62\% mencionaram ser católicas.

Em sequência, as participantes responderam ao instrumento de coleta de dados. Dos quatro domínios avaliados, o estilo de escrita obteve o maior número de respostas positivas: $97,47 \%$ das mulheres julgaram que as frases são fáceis de entender, e $98,73 \%$ que o conteúdo escrito é claro, revelando a adequação do Alertômetro ao público-alvo. O nível de concordância das respostas positivas variou de $92,41 \%$ a $98,73 \%$, resultando em uma média de $96,20 \%$, resultado suficiente para a validação do Alertômetro pela população, conforme a Tabela 3.

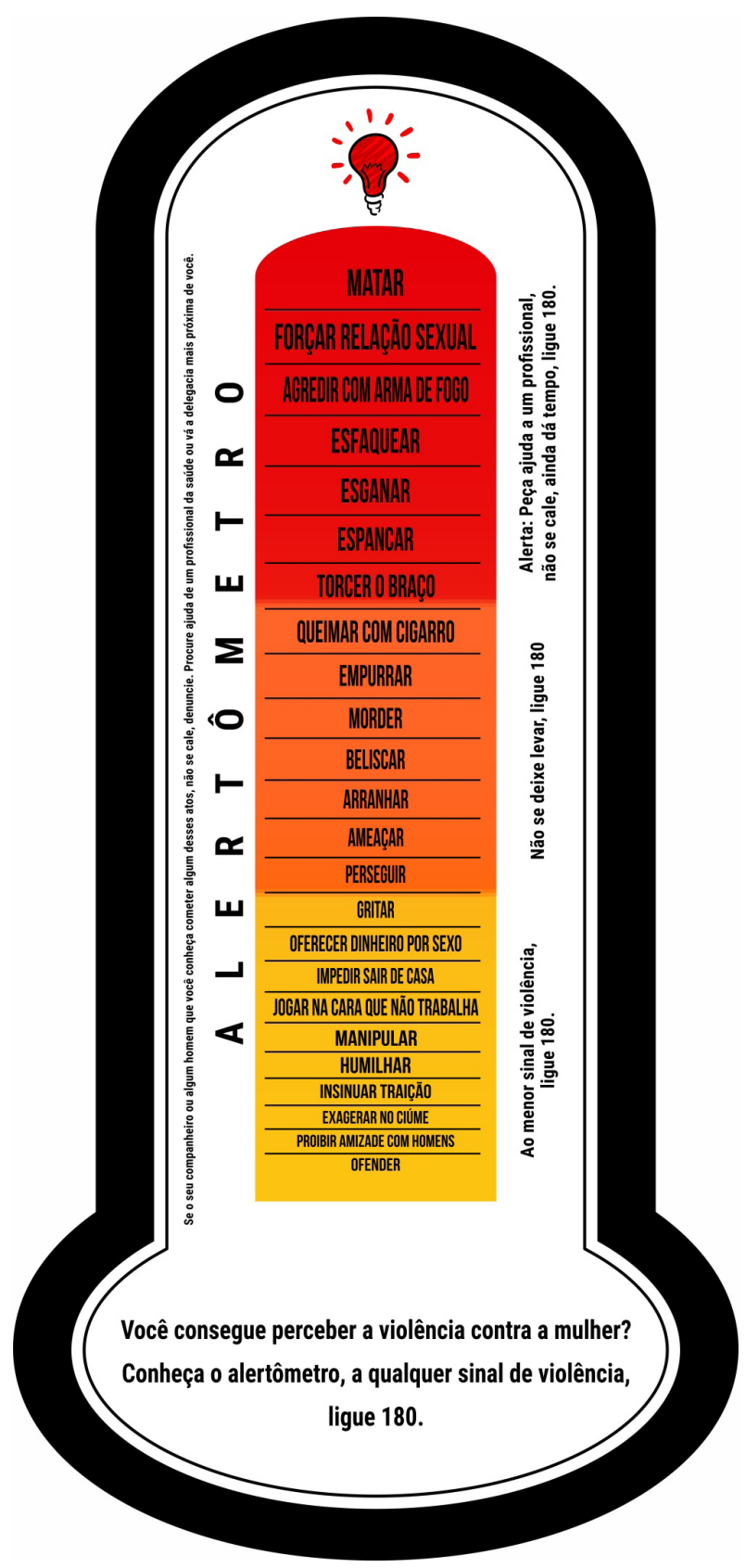

Figura 1. Versão final do Alertômetro. Teresina, Piauí, 2018.

\section{DISCUSSÃO}

De maneira geral, as respostas dos especialistas de conteúdo e técnicos foram concordantes. A partir das respostas obtidas no escore SAM, a média foi de $87,9 \%$, com nível de concordância das respostas consideradas suficientemente adequado para a validação.

Outros estudos metodológicos também analisaram seus materiais com índices suficientemente adequados: a cartilha educativa para prevenção de síndrome metabólica em adolescentes obteve SAM médio de $91,7 \%$; já a cartilha destinada à prevenção da diarreia infantil obteve $88,7 \%$ dos especialistas de conteúdo e $90,1 \%$ dos especialistas técnicos. ${ }^{16,19}$

Estudo realizado em uma policlínica universitária, envolvendo médicos, enfermeiros e estagiários, com vistas a validar uma versão em italiano do questionário produzido pela Organização Mundial da Saúde acerca da violência no ambiente de trabalho, obteve também resultados satisfatórios, boa confiabilidade e consistência interna, mostrando ser uma ferramenta útil na avaliação e prevenção da ocorrência de violência no ambiente de trabalho. ${ }^{20}$

A validação pelos especialistas de design também atingiu índices superiores relacionados ao ponto de corte empregado, sendo classificado, portanto, como adequado.

Apresentando índice relativamente superior a 0,92, pesquisadores desenvolveram em Natal-RN um curso de suporte avançado de vida à distância, alcançando um IVC global de $0,94 .{ }^{20}$ Em contrapartida, em pesquisa realizada em Fortaleza, em que se analisou uma cartilha para prevenção da transmissão vertical do HIV, atingiu-se IVC global de $0,87 .{ }^{21}$

Analisando as informações levantadas, dados semelhantes sobre validação de tecnologias educativas com índices estatísticos substanciais foram observados. Cita-se, por exemplo, a validação e a confiabilidade de uma escala, aplicada na Turquia, para avaliar a satisfação de estudantes de enfermagem referente à sua educação clínica, que teve variância de 0,93-0,99 na escala alfa de Cronbach, na qual seis fatores foram identificados, com uma variância total de $64 \%$, considerada válida e confiável. ${ }^{22}$

Por outro lado, um estudo sobre adaptação da Escala de Autotranscendência de Pamela Reed na Espanha foi validado segundo o coeficiente global de validade de conteúdo (CVC) de 0.92 , considerado de viabilidade positiva. ${ }^{23}$

Vale ressaltar que, embora validadas, ambas as escalas referidas diferem do Alertômetro quanto ao mecanismo de apresentação. O Alertômetro, por se tratar de um material dinâmico e autoexplicativo, torna-se consideravelmente aceito ao usar cores quentes para captar a atenção das mulheres, possuir uma linguagem simples e acessível tanto para o profissional, quanto para o público-alvo, e trazer um alerta das diversas manifestações da violência contra a mulher, facilitando as práticas educativas relacionadas à temática.

É indispensável enfatizar que, apesar de o Alertômetro ter sido analisado e considerado válido pelos especialistas, eles deixaram registradas suas opiniões e sugestões de modificação, 
Tabela 3. Avaliação do público-alvo quanto à organização, estilo de escrita, aparência e motivação do Alertômetro. Teresina, Piauí, Brasil, 2018

\begin{tabular}{|c|c|c|c|c|c|c|}
\hline \multirow{2}{*}{ Variáveis } & \multicolumn{2}{|c|}{ Opção 1} & \multicolumn{2}{|c|}{ Opção 2} & \multicolumn{2}{|c|}{ Opção 3} \\
\hline & $\mathrm{n}$ & $\%$ & $\mathrm{n}$ & $\%$ & $\mathrm{n}$ & $\%$ \\
\hline \multicolumn{7}{|l|}{ Organização } \\
\hline $\begin{array}{l}\text { A sequência do conteúdo está adequada? } \\
\text { (1.Sim/2.Não/3.Em parte) }\end{array}$ & 73 & 92,41 & 02 & 2,53 & 04 & 5,06 \\
\hline $\begin{array}{l}\text { A estrutura do Alertômetro está adequada? } \\
\text { (1.Sim/2.Não/3.Em parte) }\end{array}$ & 77 & 97,47 & 01 & 1,27 & 01 & 1,27 \\
\hline \multicolumn{7}{|l|}{ Estilo de escrita } \\
\hline $\begin{array}{l}\text { As frases são: } \\
\text { (1.Fáceis de entender/2.Difíceis de entender/3.Em parte) }\end{array}$ & 77 & 97,47 & 02 & 2,53 & - & - \\
\hline $\begin{array}{l}\text { As cores do Alertômetro são: } \\
\text { (1.Adequadas/2.Inadequadas/3.Em parte) }\end{array}$ & 78 & 98,73 & - & - & 01 & 1,27 \\
\hline $\begin{array}{l}\text { As ilustrações servem para facilitar o entendimento? } \\
\text { (1.Sim/2.Não/3.Em parte) }\end{array}$ & 76 & 96,20 & 01 & 1,27 & 02 & 2,53 \\
\hline \multicolumn{7}{|l|}{ Motivação } \\
\hline $\begin{array}{l}\text { Na sua opinião, qualquer mulher que ler este Alertômetro vai entender } \\
\text { do que se trata? } \\
\text { (1.Sim/2.Não/3.Em parte) }\end{array}$ & 75 & 94,94 & 02 & 2,53 & 02 & 2,53 \\
\hline
\end{tabular}

com o objetivo de garantir a melhor qualidade da tecnologia educacional a ser utilizada.

Durante o procedimento de análise do Alertômetro, as adequações realizadas, necessárias em estudos de validação, ${ }^{24}$ possibilitaram alterações que respeitassem as particularidades do público-alvo, no intuito de que o mecanismo fosse bem compreendido.

Após as sugestões de cinco especialistas, modificaram-se algumas palavras do material, com o objetivo de tornar a sua linguagem mais coloquial. À respeito do uso da linguagem, esta é considerada um fator que pode interferir de maneira significativa na compreensão da mensagem que o profissional deseja passar ao paciente. Quando não utilizada de maneira simples, há a possibilidade de que não se alcance o objetivo do material. ${ }^{25}$
Dois especialistas enfatizaram a necessidade de rever a distribuição dos tipos de violência por grau de gravidade - salientando que esse processo representa algo muito subjetivo -, em que se tem ideias diferentes do que seria violência para cada mulher. Tal observação foi considerada muito relevante, tendo sido, portanto, atendida no material adaptado.

Ademais, foi adicionado ao Alertômetro o contato do órgão ao qual recorrer em casos de violência contra a mulher, conforme alguns especialistas indicaram ser necessário. A ação conjunta da rede de atenção e apoio às mulheres vítimas de violência representa a identificação ágil e a continuidade do cuidado a essa mulher, visando atenção integral e resolutiva. ${ }^{26}$

Dessa maneira, o Alertômetro elaborado e validado no estudo se mostrou um instrumento educativo, dado o objetivo de alertar 
sobre a violência contra a mulher, considerado pela análise do público-alvo como um importante material para conscientização e disseminação de conhecimento. A participação de especialistas e representantes do público-alvo pôde elevar a credibilidade e aceitação das práticas educativas. ${ }^{27}$

A promoção de atividades educativas torna-se fundamental para o fornecimento de orientações acerca da violência contra a mulher, de forma que seja possível prevenir ou minimizar a chance de ocorrência da violência de gênero, sendo os objetos de aprendizagem ferramentas importantes para guiar e sistematizar tais ações. ${ }^{9,28}$

Os materiais educativos em saúde, quando comprovadamente validados cientificamente e empregados também de forma adequada, ou seja, inseridos e integrados no planejamento da assistência à saúde, são aliados para a construção de conhecimento em saúde. ${ }^{28,29}$

Uma vez que conceitos e condutas corretas em relação à prevenção da violência contra a mulher são devidamente preparados e veiculados para população, obtém-se, com o passar do tempo, um retorno positivo nos indicadores epidemiológicos. Sendo assim, debruçar-se sobre a tarefa de construir e validar conteúdos que conformam os materiais educativos passa ser relevante em uma sociedade que prima por diminuir seus índices de violência, cujos fatores de risco estão relacionados aos comportamentos sociais e culturais. ${ }^{29}$

Na Etiópia, pesquisadores adaptaram uma ferramenta de avaliação de violência contra a mulher em uma comunidade no Noroeste do país, com 1.269 mulheres, obtendo-se resultados satisfatórios, cujos valores foram superiores ao mínimo recomendado quanto à confiabilidade e consistência da escala adaptada. ${ }^{30}$

Analisando-se as respostas do público feminino, foi possível observar que os escores das respostas apresentaram o nível de concordância média de 96,20\%. Todavia, é necessário enfatizar o domínio motivação, cujas respostas foram as menos satisfatórias. Ou seja, a maioria das mulheres se sentiu motivada a ler o Alertômetro até o final; no entanto, um número expressivo não sentiu motivação ou a sentiu apenas em parte.

A expressão de respostas negativas das participantes pode estar relacionada ao momento da aplicação do questionário, pois a maioria das entrevistadas aguardavam suas consultas nas UBS ou referiram que precisavam retornar, o mais rápido possível, às suas residências, para começar suas atividades diárias. Por mais que os pesquisadores tenham esclarecido que a participação delas não demandaria muito tempo, foram notórios a demonstração de ansiedade e o desejo de voltar para a fila de espera ou de retornar às residências.

Apesar disso, é necessário destacar que o público-alvo avaliou positivamente o Alertômetro, considerando-o interessante, explicativo e importante. Portanto, o Alertômetro apresentou-se como uma tecnologia educacional válida na condução de discussões sobre a problemática da violência contra a mulher, o que possibilita que a mulher se aproprie das informações apresentadas, dos chamados "alertas" e do conhecimento apresentado, a fim de combater a violência no seu cotidiano.
Ressalta-se que a utilização do Alertômetro pelos profissionais de saúde será capaz de intermediar o diálogo destes com as mulheres, buscando uma produção em conjunto do conhecimento sobre a violência contra a mulher. Além disso, essa tecnologia é considerada um material que contribui para ampliar as possibilidades dos profissionais da Atenção Básica, com vistas a uma intervenção educativa que contribua com as ações dos profissionais em seu cotidiano no cuidado à mulher, possibilitando uma reflexão sobre a formação e o desenvolvimento de melhores práticas para a saúde das mulheres.

Neste contexto, a Atenção Básica se constitui como um espaço que favorece a aproximação entre os profissionais e as mulheres em situação de violência, sendo um ambiente de concretização da Política Nacional de Atenção Integral à Saúde da Mulher, para além das ações de cunho biologicistas.

\section{CONCLUSÃO E IMPLICAÇÕES PARA A PRÁTICA}

O Alertômetro elaborado mostrou-se uma tecnologia educacional válida a ser utilizada com o público feminino, pois promove conhecimento acerca das diversas manifestações da violência contra a mulher. Com relação à aplicabilidade prática, o Alertômetro poderá, no cotidiano das práticas educativas, se tornar uma tecnologia acessível tanto para os profissionais, quanto para as mulheres, com informações a serem compartilhadas de forma dinâmica e explicativa sobre as manifestações da violência contra a mulher. Consequentemente, colaborará com o processo de enfrentamento da violência.

O Alertômetro foi validado pelos especialistas de conteúdo e técnicos quanto ao conteúdo, linguagem, apresentação, estimulação/motivação e adequação cultural, obtendo escore SAM global de $87,9 \%$. Quanto à validação dos especialistas em design, no que diz respeito à aparência, resultou em IVC global de 0,92 . No que se refere à validação efetuada pelo público-alvo, houve concordância de $96,20 \%$ das respostas positivas.

Apesar de o Alertômetro ter sido validado pelos especialistas e público-alvo, o desenvolvimento do estudo apresentou como limitação metodológica o escasso referencial científico sobre validação de materiais educativos na saúde, especialmente no que tange a temática da violência contra a mulher, a fim de melhor fundamentar as análises desta pesquisa. Tal limitação não invalida os seus resultados, porém indica a necessidade de que novos estudos metodológicos sejam desenvolvidos e publicados na comunidade científica.

Considera-se que a utilização do Alertômetro poderá ser um produto de reflexão no que diz respeito ao processo educativo em saúde realizado na Atenção Básica, contribuindo para transformações de concepções e práticas dos profissionais acerca da elaboração e validação de materiais educativos sobre a violência contra a mulher, e partindo do pressuposto que o material propiciará o empoderamento feminino acerca das manifestações da violência.

Ratifica-se que as considerações aqui elencadas são direcionadas não somente ao âmbito da saúde, mas também aos profissionais que atuam na segurança pública e na justiça, pois é 
necessário um envolvimento multidisciplinar e intersetorial visando o enfrentamento da violência contra a mulher de forma holística.

\section{CONTRIBUIÇÃO DOS AUTORES}

Desenho do estudo de revisão. Aquisição, análise de dados e interpretação dos resultados. Redação e revisão crítica do manuscrito. Aprovação da versão final do artigo. Responsabilidade por todos os aspectos do conteúdo e a integridade do artigo publicado. Elayne Kelly Sepedro Sousa. Erica Jorgiana dos Santos de Morais. Camila Aparecida Pinheiro Landim Almeida. Análise de dados e interpretação dos resultados. Redação e revisão crítica do manuscrito. Aprovação da versão final do artigo. Responsabilidade por todos os aspectos do conteúdo e a integridade do artigo publicado. Fernanda Cláudia Miranda Amorim. Adélia Dalva da Silva Oliveira. Kayo Henrique Jardel Feitosa Sousa.

\section{EDITOR ASSOCIADO}

\author{
Cândida Caniçali Primo
}

\section{REFERÊNCIAS}

1. Cortes LF, Padoin SMM, Vieira LB, Landerdahl MC, Arboit J. Care for women victims of violence: empowering nurses in the pursuit of gender equity. Rev Gaúcha Enferm. 2015;36(Spec No):77-84. http://dx.doi. org/10.1590/1983-1447.2015.esp.57162. PMid:27057705.

2. Santos WJ, Oliveira PP, Viegas SMF, Ramos TM, Policarpo AG, Silveira EAA. Domestic violence against women perpetrated by intimate partner: professionals' social representations in primary health care. Rev Fund Care Online. 2018;10(3):770-7. http://dx.doi.org/10.9789/2175-5361.2018. v10i3.770-777.

3. Broch D, Crossetti MGO, Riquinho DL. Reflections on violence against women in the perspective of Madeleine Leininger. Rev Enferm UFPE. 2017;11(12):5079-84. http://dx.doi.org/10.5205/1981-8963v11i12a22588p5079-5084-2017.

4. Sardinha LM, Catalán HEN. Attitudes towards domestic violence in 49 low- and middle-income countries: a gendered analysis of prevalence and countrylevel correlates. PLoS One. 2018;13(10):e0206101. http:// dx.doi.org/10.1371/journal.pone.0206101. PMid:30379891.

5. Chuemchit M, Chernkwanma S, Rugkua R, Daengthern L, Abdullakasim P, Wieringa AE. Prevalence of intimate partner violence in Thailand. J Fam Violence. 2018;33(5):315-23. http://dx.doi.org/10.1007/s10896018-9960-9. PMid:29904232.

6. Silva NNF, Leal SMC, Trentin D, Vargas MAO, Vargas CP, Vieira LB. Atuação dos enfermeiros da atenção básica a mulheres em situação de violência. Enferm. Foco. 2017;8(3):70-4. http://dx.doi.org/10.21675/2357707X.2017.v8.n3.1290.

7. Rodrigues WFG, Rodrigues RFG, Ferreira FA. Violence against women within a biopsyocial context: a challenge for the nursing professional. Rev Enferm UFPE Online. 2017;11(4):1752-8. http://dx.doi.org/10.5205/19818963-v11i4a15247p1752-1758-2017.

8. Neves VNS, Prestes EMT, Sabino RN, Silva MLN, Barros AG. Four pillars of education for the twenty-first century in the continuing education of health professionals. Rev Enferm UFPE Online. 2016;10(4):3524-30.

9. Polit DF, Hungler BP. Fundamentos de pesquisa em enfermagem. $4^{\mathrm{a}}$ ed. Porto Alegre: Artes Médicas; 2000.

10. Polit DF, Beck CT. Fundamentos de pesquisa em enfermagem: avaliação de evidências para as práticas da Enfermagem. $7^{\text {a }}$ ed. Porto Alegre: Artmed; 2011.669 p.

11. Salvador PTCO, Mariz CMS, Vitor AF, Ferreira MA Jr, Fernandes MID, Martins JCA et al. Validation of virtual learning object to support the teaching of nursing care systematization. Rev Bras Enferm. 2018;71(1):1624. http://dx.doi.org/10.1590/0034-7167-2016-0537. PMid:29324939.
12. Teles LMR, Oliveira AS, Campos FC, Lima TM, Costa CC, Gomes LFS et al. Development and validating an educational booklet for childbirth companions. Rev Esc Enferm USP. 2014;48(6):977-84. http:// dx.doi.org/10.1590/S0080-623420140000700003. PMid:25626495.

13. Freitas LV, Teles LMR, Lima TM, Vieira NFC, Barbosa RCM, Pinheiro $A K B$ et al. Exame físico no pré-natal: construção e validação de hipermídia educativa para a Enfermagem. Acta Paul Enferm. 2012;25(4):581-8. http://dx.doi.org/10.1590/S0103-21002012000400016.

14. Hill MM, Hill A. Investigação por questionário. $2^{\underline{a}}$ ed. Lisboa: Sílabo; 2012.

15. Doak CC, Doak LG, Root JH. Teaching patients with low literacy skills. 2nd ed. Philadelphia: J.B. Lippincott; 1996. 212 p.

16. Moura IH, Silva AFR, Rocha AESH, Lima LHO, Moreira TMM, Silva ARV. Construction and validation of educational materials for the prevention of metabolic syndrome in adolescents. Rev Latino-Am. 2017;25(0):e2934. http://dx.doi.org/10.1590/1518-8345.2024.2934. PMid:29020125.

17. Alexandre NMC, Coluci MZO. Content validity in the development and adaptation processes of measurement instruments. Cien Saude Colet. 2011;16(7):3061-8. http://dx.doi.org/10.1590/S1413-81232011000800006 PMid:21808894.

18. Polit DF, Beck CT. The content validity index: are you sure you know what's being reported? Critique and recommendations. Res Nurs Health. 2006;29(5):489-97. http://dx.doi.org/10.1002/nur.20147. PMid:16977646.

19. Sabino LMM, Ferreira ÁMV, Joventino ES, Lima FET, Penha JC, Lima KF et al. Elaboration and validation of a reader on childhood diarrhea prevention. Acta Paul Enferm. 2018;31(3):233-9. http://dx.doi. org/10.1590/1982-0194201800034.

20. La Torre G, Sestili C, lavazzo E, Mannocci A. Fenomeno violenza súbita sul luogo di lavoro nel settore sanitário: validazione della versione italiana del questionario WHO. Clin Ter. 2017;168(3):e199-202. http:// dx.doi.org/10.7417/T.2017.2006. PMid:28612897.

21. Costa IKF, Tibúrcio MP, Melo GSM, Leite JEL, Dantas RAN, Torres GV. Construction and validation of a distance Basic Life Support Course. Rev Bras Enferm. 2018;71(Suppl 6):2698-66. http://dx.doi. org/10.1590/0034-7167-2018-0122. PMid:30540046.

22. Lima ACMACC, Bezerra KC, Sousa DMN, Rocha JF, Oriá MOB Development and validation of a booklet for prevention of vertical HIV transmission. Acta Paul Enferm. 2017;30(2):181-9. http://dx.doi. org/10.1590/1982-0194201700028.

23. Atay S, Kurt FY, Aslan GK, Saarikoski M, Yilmaz H, Ekinci V. Validity and reliability of the Clinical Learning Environment, Supervision and Nurse Teacher (CLES+T), Turkish version. Rev Lat Am Enfermagem. 2018;26(0):e3037. http://dx.doi.org/10.1590/1518-8345.2413.3037. PMid:30208159.

24. Pena-Gayo A, González-Chordá VM, Cervera-Gasch A, Mena-Tudela D. Cross-cultural adaptation and validation of Pamela Reed's SelfTranscendence Scale for the Spanish context. Rev Lat Am Enfermagem. 2018;26(0):e3058. http://dx.doi.org/10.1590/1518-8345.2750.3058. PMid:30540116.

25. Nunes LB, Almeida CAPL, Rodrigues MTP, Lago EC, Moura LKB, Coêlho MCVS. Cross-cultural adaptation of a tool to assess therapeutic educational interventions for diabetes patients. Cogitare Enferm. 2018;23(3):e55337. http://dx.doi.org/10.5380/ce.v23i3.55337.

26. Farias MS, Ponte KMA, Gomes DF, Menezes RSP. Educational technology on gastric cancer. Rev Enferm UFPE Online. 2018;12(4):947-52. http:// dx.doi.org/10.5205/1981-8963-v12i4a230434p947-952-2018.

27. Cortes LF, Padoin SM, Kinalski DDF. Instruments for articulating the network of attention to women in situation of violence: collective construction. Rev Gaúcha Enferm. 2017;37(spe):e20160056. PMid:28640334.

28. Pereira AC, Soares VL, Russo TMS, Teles AAS, Lenza NFB, Sonobe HM. Pre-operative education in the perspective of cancer patients. Rev Enferm UDPE. 2016;10(2):449-56. http://dx.doi.org/10.1590/00347167-2016-0622.

29. Antunes ML. A literacia em saúde: investimento na promoção da saúde e na racionalização de custos. Actas das XI Jornadas APDIS: As Bibliotecas da Saúde, que Futuro?; 2014; Lisboa. Lisboa: APDIS; 2014. p. 123-33.

30. Semahegn A, Torpey K, Manu A, Assefa N, Ankomah A. Adapted tool for the assessment of domestic violence against women in a low-income country setting: a reliability analysis. Int J Womens Health. 2019;11(11):6573. http://dx.doi.org/10.2147//JWH.S181385. PMid:30774451. 